\title{
The Targeting of Plasmalemmal Ceramide to Mitochondria during Apoptosis
}

\author{
Eduard B. Babiychuk, Alexander P. Atanassoff, Katia Monastyrskaya ${ }^{\text {aa }}$, Christina Brandenberger ${ }^{\text {ab }}$, \\ Daniel Studer, Catherine Allemann, Annette Draeger*
}

Institute of Anatomy, University of Bern, Bern, Switzerland

\begin{abstract}
Ceramide is a key lipid mediator of cellular processes such as differentiation, proliferation, growth arrest and apoptosis. During apoptosis, ceramide is produced within the plasma membrane. Although recent data suggest that the generation of intracellular ceramide increases mitochondrial permeability, the source of mitochondrial ceramide remains unknown. Here, we determine whether a stress-mediated plasmalemmal pool of ceramide might become available to the mitochondria of apoptotic cells. We have previously established annexin $\mathrm{A} 1-\mathrm{a}$ member of a family of $\mathrm{Ca}^{2+}$ and membrane-binding proteins - to be a marker of ceramide platforms. Using fluorescently tagged annexin A1, we show that, upon its generation within the plasma membrane, ceramide self-associates into platforms that subsequently invaginate and fuse with mitochondria. An accumulation of ceramide within the mitochondria of apoptotic cells was also confirmed using a ceramide-specific antibody. Electron microscopic tomography confirmed that upon the formation of ceramide platforms, the invaginated regions of the plasma membrane extend deep into the cytoplasm forming direct physical contacts with mitochondrial outer membranes. Ceramide might thus be directly transferred from the plasma membrane to the mitochondrial outer membrane. It is conceivable that this "kiss-of-death" increases the permeability of the mitochondrial outer membrane thereby triggering apoptosis.
\end{abstract}

Citation: Babiychuk EB, Atanassoff AP, Monastyrskaya K, Brandenberger C, Studer D, et al. (2011) The Targeting of Plasmalemmal Ceramide to Mitochondria during Apoptosis. PLoS ONE 6(8): e23706. doi:10.1371/journal.pone.0023706

Editor: Orian S. Shirihai, Boston University, United States of America

Received January 17, 2011; Accepted July 22, 2011; Published August 19, 2011

Copyright: (c) 2011 Babiychuk et al. This is an open-access article distributed under the terms of the Creative Commons Attribution License, which permits unrestricted use, distribution, and reproduction in any medium, provided the original author and source are credited.

Funding: The study was supported by grants from the Swiss National Science Foundation http://www.snf.ch/D/Seiten/default.aspx to AD (320030_128064/1), EB (3100A0-121980/1) and KM (320000-111778). The funders had no role in study design, data collection and analysis, decision to publish, or preparation of the manuscript.

Competing Interests: The authors have declared that no competing interests exist.

* E-mail: draeger@ana.unibe.ch

aa Current address: Urology Research Laboratory, Department Clinical Research, University of Bern, Bern, Switzerland

ab Current address: Lung Biology Laboratory, Department of Medicine, Columbia University, New York, United States of America

\section{Introduction}

An increased permeability of the mitochondrial outer membrane is implicated in the early stages of apoptosis [1]. The release of pro-apototic proteins from mitochondria can be mediated by the activation of Bax [2]. Recent data suggest that Bax preferentially inserts into mitochondria via ceramide-enriched microdomains, however, details of its targeting mechanism have not been resolved [3-5]. On the other hand, an increase in mitochondrial permeability has been ascribed to ceramide itself: Accumulation of mitochondrial ceramide might promote the formation of membrane channels and the subsequent release of proapoptotic proteins from the mitochondrial intermembrane space [6].

Mitochondria do not participate in interorganellar vesicular trafficking. Hence, during the onset of apoptosis, ceramide has to be either produced on-site or rapidly transported to the mitochondria from other sources. Sphingomyelinase-dependent hydrolysis of sphingomyelin and de novo synthesis are the two major pathways of ceramide biosynthesis [7-9]. The de novo production of ceramide is confined to the endoplasmic reticulum, and the mechanism whereby the newly synthesized ceramide is transferred to the mitochondria is unknown. A recent study has provided evidence for the existence of a mitochondrial-associated neutral sphingomyelinase [10]. It is thus possible that mitochondrial sphingolipids $[11,12]$ are metabolically converted to ceramide in situ and are locally assembled into ceramide channels within the mitochondrial outer membrane $[13,14]$. However, it is particularly the plasmalemmal ceramide, which is generated by the activity of acid sphingomyelinase that is implicated in the induction of apoptosis [14]. Moreover, a soluble ceramide transfer protein (CERT) has been described [15], which could transport ceramide to the mitochodria from the Golgi apparatus. Thus, although an increase in mitochondrial ceramide in response to the initiation of apoptosis has been documented [4,16], the source of mitochondrial ceramide and its mechanism of entry are unknown.

Massive, calcium-activated endocytosis of the plasma membrane without involvement of classical endocytic proteins is a recently described phenomenon and is closely associated with the formation of ceramide during cellular stress [17-20]. We have investigated interorganellar membrane contacts during this process.

Using confocal microscopy in living cells and electron microscopic tomography of unfixed, high-pressure frozen cells, we have demonstrated that ceramide platforms, initially formed within the plasma membrane of apoptotic cells, are internalized and come into close contact with the mitochondrial outer membrane. It is conceivable that ceramide is exchanged at these 
contact points, and that this triggers the release of pro-apoptotic molecules.

\section{Materials and Methods}

\section{Reagents and antibodies}

The mouse polyclonal antibody against ceramide [21] was purchased from Glycobiotech $\mathrm{GmbH}$ (Kuekels, Germany). The Living Colours Fluorescent protein vectors peCFP-N1, peYFP-N1 and pDsRed-Mito were obtained from Clontech Europe ( $\mathrm{St}$ Germain-en-Laye, France), and the restriction endonucleases, Taq polymerase and T4 DNA ligase from New England Biolabs (Bioconcept, Allschwil, Switzerland). Other reagents were purchased from Sigma (Buchs, Switzerland).

\section{Cell culture and transfections}

The coding sequences of annexins Al and A6 were cloned into the Living Colours Fluorescent protein vectors yellow-fluorescent protein (YFP), green fluorescent protein (GFP) and cyan fluorescent protein (CFP), following the PCR amplification from human bladder smooth muscle cDNA [22].

Jurkat $\mathrm{T}$ cells and a human monocyte cell line (THP-1) were cultured in RPMI medium containing $5 \%$ calf serum and penicillin/streptomycin. The cells were grown in $5 \% \mathrm{CO}_{2}$ at $37^{\circ} \mathrm{C}$ in a humidified incubator. They were transiently transfected with plasmids by electroporation (BioRad) and analysed after incubation at $37^{\circ} \mathrm{C}$ for 48 hours.

\section{Confocal imaging}

Annexin Al-YFP, annexin A1-GFP, annexin A6-CFP and dsRed-Mito were transiently expressed in Jurkat T-cells and THP1 cells. The cells, which were allowed to settle on glass coverslips, were mounted in a perfusion chamber in Tyrode's solution (140 mM NaCl, $5 \mathrm{mM} \mathrm{KCl,} 1 \mathrm{mM} \mathrm{MgCl}_{2}, 10 \mathrm{mM}$ glucose, $10 \mathrm{mM}$ HEPES, $\mathrm{pH}=7.4$ ) to which was added $2 \mathrm{mM} \mathrm{CaCl}_{2}$. At time point zero, the cells were challenged either with Streptolysin O $[$ SLO $(100 \mathrm{ng} / \mathrm{ml})]$ or with ionomycin $(5 \mu \mathrm{M})$. The fluorescence signal was recorded using a $\times 100$ oil immersion lens in an Axiovert $200 \mathrm{M}$ microscope with a laser scanning module LSM 510 META (Zeiss, Germany).

\section{Immunofluorescence analysis}

Immunofluorescence was performed as previously described $[22,23]$, with the following modifications for non-adherent cells: Following centrifugation at $100 \mathrm{~g}$, the pelleted Jurkat $\mathrm{T}$ and the THP-1 cells were suspendend in Tyrode's solution. Some cells were treated with a cell permeant, mitochondrium-specific dye [Mito-ID $^{\mathrm{TM}}$ Red (Enzo Life Sciences, Lausen, Switzerland)] according to the manufactorer's instructions, followed by SLO $(100 \mathrm{ng} / \mathrm{ml})$ or ionomycin $(5 \mu \mathrm{M})$ treatment in suspension in Tyrode's buffer containing $2 \mathrm{mM} \mathrm{Ca}^{2+}$ for $15 \mathrm{~min}$. The cells were allowed to settle on coverslips, chemically fixed with $4 \%$ paraformaldehyde in Tyrode's solution for $5 \mathrm{~min}$ and finally permeabilized with $0.5 \%$ Triton X-100 (in Tyrode's solution) for $30 \mathrm{~s}$ at ambient temperature.

The colocalization of mitochondria with ceramide reporter protein annexin A1-GFP after stimulation of cells with ionomycin was quantified in Jurkat cells in 4 independent experiments and with SLO in 2 independent experiments. Identical stimulations were conducted in THP-1 cells with ionomycin ( 5 independent experiments) and with SLO (2 independent experiments). The percentage of colocalization of annexin Al-GFP with Mito-ID ${ }^{\mathrm{TM}}$ Red in each cell was established by calculating the ratio of merged pixels (red/green) to the total pixels, showing a fluorescent intensity in either of the channels above the background, within the observation field containing at least 30 cells.

\section{Ultrastructural investigations}

$\mathrm{G}_{\mathrm{m} 1}$ ganglioside receptors on the plasmalemmal surface of Jurkat T-cells and THP-1 cells were labelled with horseradishperoxidase-tagged cholera toxin B (HRP-CTX B), and then stimulated either with SLO $(100 \mathrm{ng} / \mathrm{ml})$ or with ionomycin $(5 \mu \mathrm{M})$ for $20 \mathrm{~min}$. The peroxidase was developed with $0.02 \%$ diaminobenzidine in Tyrode's buffer containing $2 \mathrm{mM} \mathrm{Ca}^{2+}$ and $0.01 \%$ $\mathrm{H}_{2} \mathrm{O}_{2}$ for $15 \mathrm{~min}$. The samples were washed in Tyrode's solution containing $2 \mathrm{mM} \mathrm{CaCl}_{2}$ and the samples were immediately subjected to a pressure of $210 \mathrm{MPa}$ [using a Leica EMPACT instrument (FEI Company, The Netherlands)] and simultaneously cooled down to $-196^{\circ} \mathrm{C}$ by a double jet of liquid nitrogen [24]. Freeze substitution was performed as described previously [25]. In brief, the samples were dehydrated at $-90^{\circ} \mathrm{C}$, treated with $2 \%$ osmium tetroxide at $-70^{\circ} \mathrm{C}$ and embedded in Epon at temperatures between $-50^{\circ} \mathrm{C}$ and ambient temperature. $250 \mathrm{~nm}$-thick-sections were subjected to electron microscopic tomography in a Tecnai F20 transmission electron microscope, which was equipped with a GIF Tridiem energy filter and an Ultrascan 1000 CGD camera (Gatan, Pleasanton, USA). The samples were secured in a dual tilt Fischione specimen holder (Fischione Instruments, USA) and tomograms were recorded continuously over a tilt-angle shift ranging $-70^{\circ}$ to $+70^{\circ}$. To correct for the missing wedge $\left(-90^{\circ}\right.$ to $-70^{\circ} \%+90^{\circ}$ to $\left.+70^{\circ}\right)$ dual tilt axis acquisition was performed with an angle difference of $90^{\circ}$. Image processing and 3D-stack reconstruction were achieved using the Inspect 3D software V.3.0. (FEI Company).

Plasmalemmal invaginations were counted in ultrathin sections of Jurkat cells $(n=1800)$ in 6 independent experiments $(\sim 25$ randomly selected visual fields/experiment). Plasmalemmal-mitochondrial proximity was considered evident at $\leq 0.2 \mu \mathrm{m}$ distance between the 2 organelles.

\section{Results}

\section{Intracellular $\mathrm{Ca}^{2+}$-overload induces an accumulation of mitochondrial ceramide}

$\mathrm{Ca}^{2+}$-overload is a potent pro-apoptotic stimulus [26,27], which leads to the hydrolysis of sphingomyelin and the formation of ceramide at the plasma membrane [18].

The observation that ceramide was present within mitochondria during the early stages of apoptosis [28] prompted us to investigate a potential transport of ceramide from the plasmalemma to mitochondria.

An intracellular $\mathrm{Ca}^{2+}$-overload was induced in Jurkat T-cells or in THP-1 cells by treating them with the pore-forming toxin SLO or with ionomycin [18]. Immunofluorescence microscopy of cells permeabilized with SLO revealed a partial colocalization of the mitochondrial marker dsRed-Mito with a monospecific antibody against ceramide (Fig. 1). No ceramide was detected within unstimulated (control) cells (Fig. 1).

Similarly, a partial colocalization between mitochondria (labelled with Mito-ID ${ }^{\mathrm{TM}} \mathrm{Red}$ ) and ceramide, labelled with the ceramide-reporter protein annexin Al [18,29]), was observed (Fig. 2, arrows). Within unstimulated (control) cells, annexin A1GFP was diffusely distributed throughout the cytoplasm (Fig. 2).

Colocalization analysis revealed $29 \pm 10.8 \%$ overlap of MitoID $^{\mathrm{TM}}$ Red and annexin Al-GFP in Jurkat cells (ionomycin: $\mathrm{n}=24$; 4 independent experiments, SLO: $\mathrm{n}=13$; 2 independent experiments) and $31 \pm 17.8 \%$ STD overlap in THP-1 cells (ionomycin: 

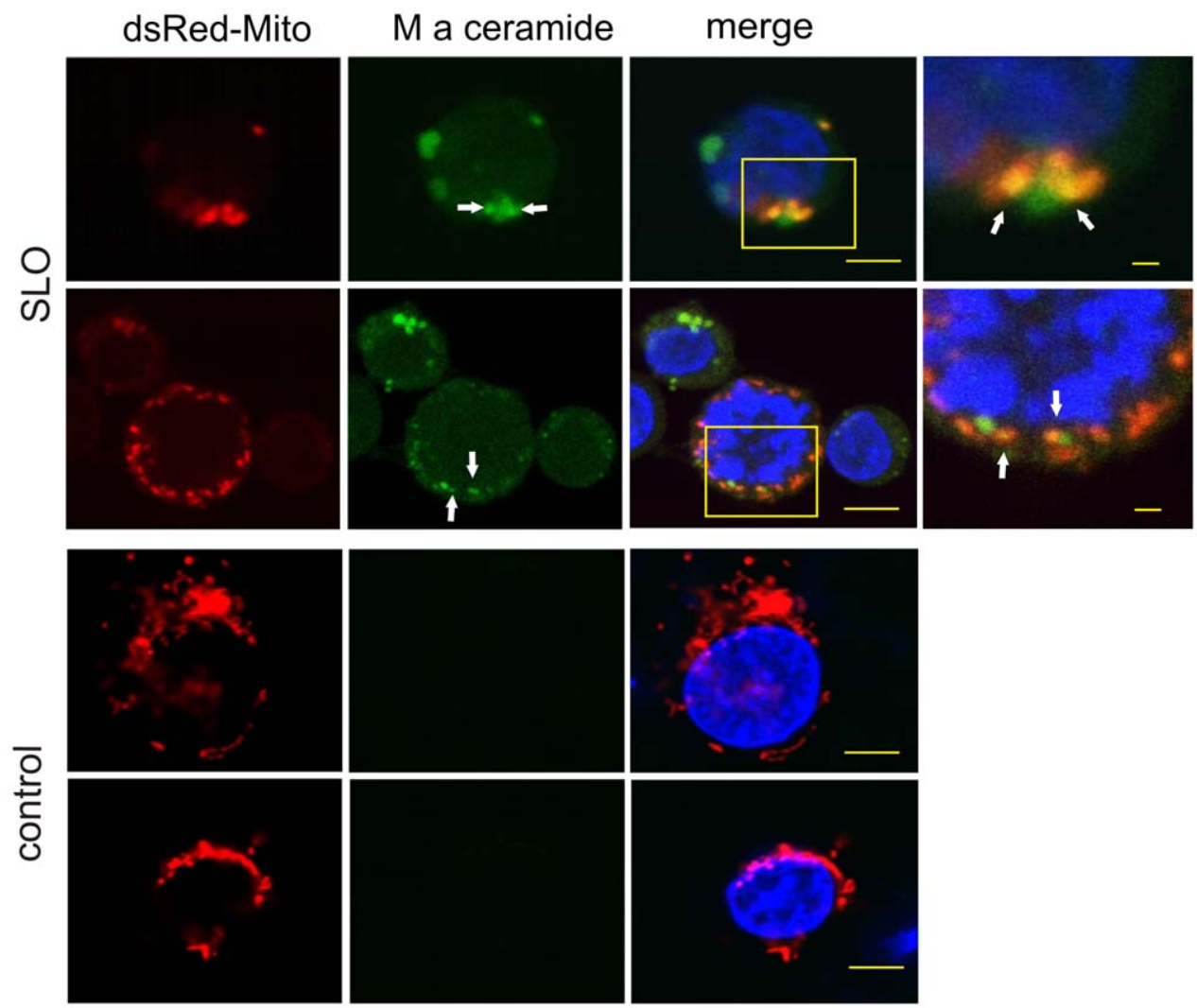

Figure 1. $\mathrm{Ca}^{2+}$-overload leads to the appearance of ceramide in mitochondria. The mitochondrial marker dsRed-Mito (red) was transiently expressed in Jurkat T-cells, which were either stimulated with SLO or left untreated (control). Subsequently, the cells were chemically fixed and treated with a polyclonal antibody against ceramide ( $\mathrm{M}$ a ceramide; green) and Hoechst stain as a nuclear marker (blue). Confocal micrographs revealed ceramide platforms and their partial colocalization with mitochondria in stimulated cells (yellow, arrows). The boxed areas are enlarged. No ceramide platforms are present in unstimulated cells. Bars in the merged images $=3 \mu \mathrm{m}$, enlarged boxed areas $=1 \mu \mathrm{m}$. doi:10.1371/journal.pone.0023706.g001

$\mathrm{n}=19 ; 5$ independent experiments, SLO: $\mathrm{n}=9 ; 2$ independent experiments).

\section{Internalisation of plasmalemmal ceramide platforms}

Upon persistent intracellular $\mathrm{Ca}^{2+}$-elevation, annexin $\mathrm{Al}$ associates with ceramide platforms, while annexin A6 delineates the plasma membrane due to their differential lipid sensitivity of membrane binding [18,22,29].

Real-time confocal microscopy of Jurkat T-cells, that had been doubly transfected with annexin Al-YFP and annexin A6-CFP shows that an increase in $\left[\mathrm{Ca}^{2+}\right]_{i}$ led to the translocation of both annexins from the cytoplasm to the plasma membrane (Fig. 3). Initially, homogenously co-distributed, the two annexins began to segregate as the formation and self-association of ceramide progressed (Fig. 3: 134s). The accumulation of ceramide/annexin Al platforms was followed by extensive invagination of the plasmalemma (Fig. 3: 134-150s) and their translocation from the plasma membrane to the interior of the cell (Fig. 3 \& Movie S1). Annexin A6 did not participate in platform formation and was not internalised (Fig. 3 \& Movie S1).

\section{Physical interaction between the invaginated plasma} membrane and the mitochondria visualized by electron microscopic tomography

In search of a potential physical interaction between the invaginated plasma membrane and the mitochondria, the process of invagination was monitored by electron microscopy. Since lipids are not immobilised by chemical fixation, preparation artefacts can distort lipid-driven changes in membrane curvature. In order to preserve the ultrastructure of the membranes in a near-native state, the molecules were immobilised by high-pressure freezing $(210 \mathrm{MPa})$ with cooling rates which avert the formation and growth of intracellular ice crystals [24]. This process was followed by freeze substitution, which, in contrast to chemical fixation in a liquid phase, preserves the 3D framework of the constituent molecules in the absence of osmotic effects (Fig. 4). These methods combined high-speed immobilisation and preservation of cellular structures without chemical cross-linking. Thus, the ultrastructural pattern of "native" membrane internalisation could be investigated. Consistent with the lower-resolution confocal images (Fig. 3), resting Jurkat T-cells (Fig. 4a) or THP-1 cells (not shown) displayed a uniformal, smooth plasmalemmal surface. In cells, that had been exposed to a $\mathrm{Ca}^{2+}$-overload, tube-like invaginations were observed, branching out from numerous plasmalemmal sites and extending deep into the intracellular space (Fig. 4b boxed area enlarged in d).

An inhibitor of acid sphingomyelinase (desipramine; [30]), was used to reduce the levels of ceramide after $\left[\mathrm{Ca}^{2+}\right]_{\mathrm{i}}$ overload [18]. Cells treated with $50 \mu \mathrm{M}$ desipramine for $90 \mathrm{~min}$ prior to exposure to SLO showed a clumpy cytoplasm and engorged intracellular organelles, but did not display plasmalemmal infoldings or disruptions (Fig. 4c, see also [18]).

To facilitate the structural identification of the sites of membrane invagination, the plasmalemmal surface was pre- 


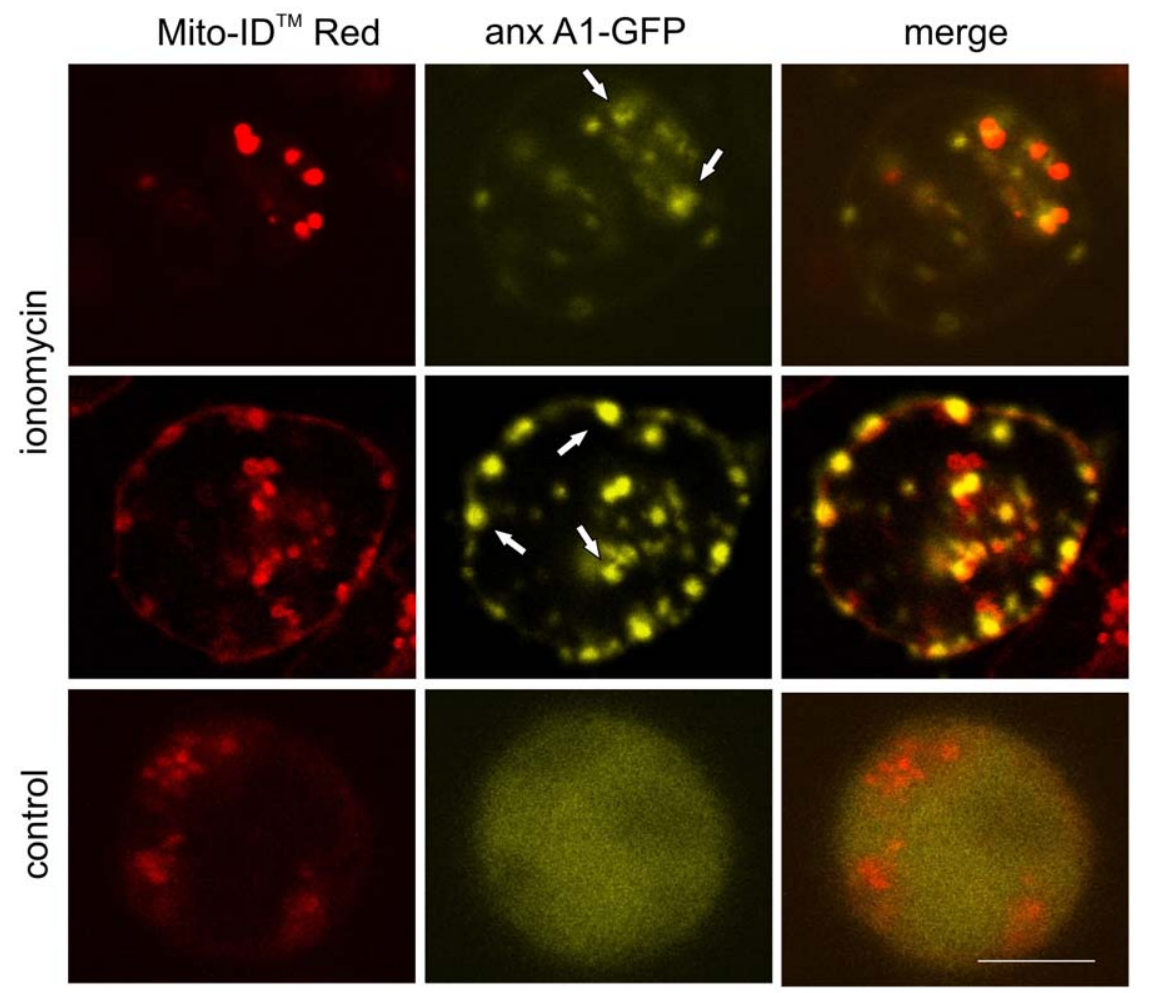

Figure 2. Annexin A1 partially colocalises with mitochondria after intracellular $\mathrm{Ca}^{2+}$-overload. Confocal micrographs of living Jurkat $\mathrm{T}$ cells that had been transiently transfected with annexin A1-GFP, treated with the mitochondrial marker Mito-ID ${ }^{T M}$ Red (red) and stimulated with ionomycin. A partial co-localization of annexin A1 with mitochondria can be observed (arrows). The untreated cell (control) shows a homogeneous distribution of annexin $\mathrm{A} 1$ throughout the cytoplasm. Bar $=3 \mu \mathrm{m}$. doi:10.1371/journal.pone.0023706.g002

labelled with the horseradish-peroxidase-tagged cholera toxin subunit B. Some of the horseradish-peroxidase-tagged, tube-like structures emanated from the plasma membrane, extended deep into the cytoplasm (Fig. 4e, boxed area enlarged in $4 \mathrm{f}$, arrowhead) and ended in the vicinity of a mitochondrium (Fig. 4f, arrow). Electron microscopic tomography of $250 \mathrm{~nm}$-thick, uncontrasted sections permitted a 3D-reconstruction of the invaginated plasmalemmal components (Fig. 5 arrowheads) which were observed to directly contact the mitochondrial outer membranes (Fig. 5 arrows, Movies S2 and S3). Plasmalemmal invaginations were found in $37.5 \%( \pm 12 \% \mathrm{STD})$ of Jurkat cells in which $\mathrm{Ca}^{2+}$ overload had been elicited ( $n=1800$ of 6 independent experiments). Close proximity of plasmalemma and mitochondria (a distance of $\leq 0.2 \mu \mathrm{m}$ between the plasmalemma and the outer mitochondrial membrane) was observed in $4.1 \%( \pm 2.6 \%$ STD $)$ of Jurkat cells. The distance of $200 \mathrm{~nm}$ was selected on the basis of micrographic tomography experiments that demonstrated that this distance was highly likely to lead to a direct contact and a physical apposition of the different membranes in the sections above or below the sectioned plane.

\section{Discussion}

Apoptosis can be triggered by a receptor-mediated, extrinsic or by an intrinsic pathway. Both pathways are thought to be interlinked and converge on the mitochondria, whose outer membranes become increasingly permeable [31]. Consequently, proteins leaking out from the intermembranous space activate cytosolic caspases and DNAses, which ultimately lead to apoptotic cell death [32]. This increase in the permeability of the mitochondrial outer membrane has been ascribed to proteins and lipids alike. Firstly, the release of pro-apototic proteins from mitochondria can be mediated by Bax [1] which translocates to the mitochondria from the cytosol $[3,4]$. Secondly, the increase in permeability occurs due to a local action of ceramide $[6,13,33]$. However, the mechanism by which ceramide appears in the mitochondrial membrane is not unequivocally established. Intriguingly, ceramide that is generated by the activity of acid sphingomyelinase within the plasma membrane is considered to be crucial for the regulation of apoptosis that is triggered by the activation of death receptors, cytotoxic drugs and environmental stress stimuli $[14,34,35]$. The central role of the plasmalemmal pool of ceramide (ceramide platforms) in the induction of apoptosis has been demonstrated in acid sphingomyelinase(ASM) deficient cells and in ASM-deficient mice [36-38]. New data identify ceramide platforms as nucleation sites and import vehicles for cationic cell-penetrating peptides [39].

We have previously shown that plasmalemmal ceramide production, its self-association to membrane platforms and platform invagination/internalization is critically dependent on the elevation of intracellular $\mathrm{Ca}^{2+}$. No ceramide platform formation/invagination was observed in the presence of EGTA [18].

Here, we ascertained whether a stress-mediated plasmalemmal pool of ceramide might become available to the mitochondria of apoptotic cells. Using immunofluorescent microscopy we have confirmed an accumulation of ceramide within the mitochondria of fixed apoptotic cells. Using annexin Al as a marker for ceramide platforms [18], we investigated structural changes in the plasma membrane of living cells undergoing $\mathrm{Ca}^{2+}$-overload- 

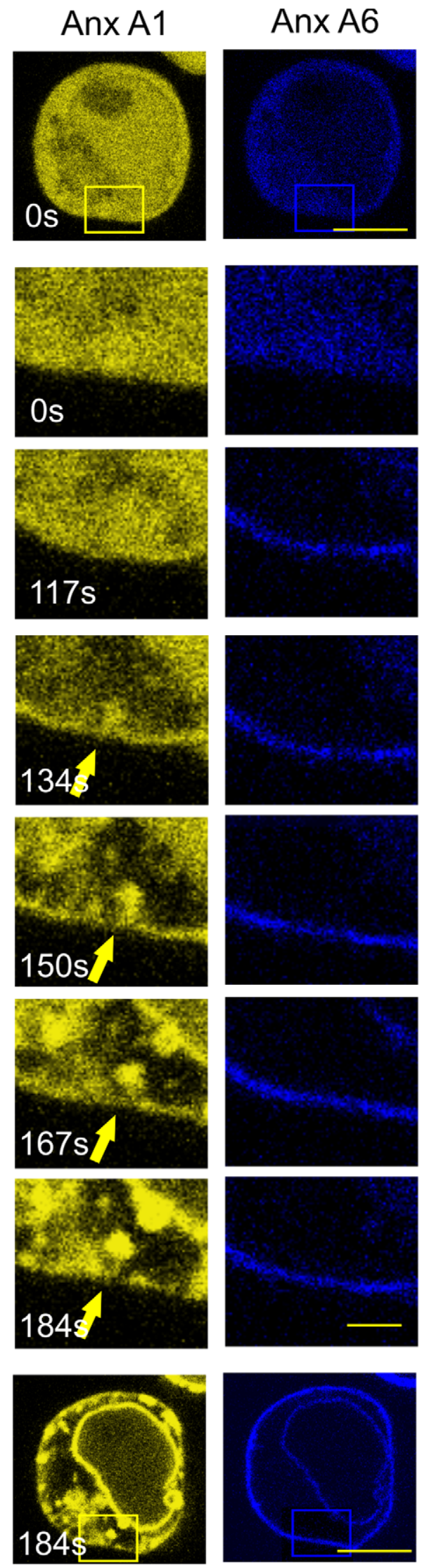

\section{Time after SLO addition (s)}

Figure 3. Annexin A1 invagination in a Jurkat T-cell after intracellular $\mathrm{Ca}^{2+}$-overload. Time-lapse sequence of confocal micrographs for a Jurkat T-cell that had been transiently transfected with annexin A1-YFP (Anx A1, yellow) and annexin A6 (Anx A6, blue) prior to stimulation with SLO. Both annexins translocate to the plasma membrane between time points 117-134. Thereafter, annexin A1 segregates from annexin A6 and coalesces into membrane platforms, which are internalised. The development of annexin A1-decorated, finger-like invaginations after $\mathrm{Ca}^{2+}$ overload (arrows) was monitored over 3 min (time in s). Images at selected time-points are illustrated. Those at time-points zero and $184 \mathrm{~s}$ (bars $=3 \mu \mathrm{m}$ ) are represented at lower magnification (boxed areas; $\mathrm{bar}=1 \mu \mathrm{m}$ ) to aid orientation. doi:10.1371/journal.pone.0023706.g003

induced apoptosis. Extensive internalisation of the plasmalemmal annexin Al/ceramide platforms was observed and the invaginated plasma membrane established contacts with the mitochondrial outer membrane.

The most conclusive evidence for the establishment of direct contacts between the ceramide-rich plasmalemmal invaginations and mitochondria came from observations in the electron microscope. Fine structural analyses of dynamic membrane compartments are technically challenging. The preservation of cells by high-pressure freezing and freeze substitution permits a stabilisation of transient cell structures $[24,25]$ which cannot be achieved by chemical fixation. Using this technique, it has become possible to characterize more precisely not only major cytoskeletal structures, but also membranous systems and their dynamic relationships [40]. Labelling of the plasmalemmal surface permits an identification and visual tracing of internalized membranes. Our initial analysis of individual sections, revealed the apoptotic cells to display numerous, closed plasmalemma-derived vesicles. The generation of plasmalemmal infoldings and vesiculation is prevented by an inhibitor of acid sphingomyelinase, desipramine. But the full extent of the tubular invaginations was disclosed only by $3 \mathrm{D}$ electron microscopic tomography. Our data indicate that the vesicles comprise a communicating network of plasmalemmal invaginations which are rapidly formed in response to $\mathrm{Ca}^{2+}$ overload. And most importantly, these structures establish direct physical contacts with the mitochondrial outer membranes.

Massive, calcium-activated endocytosis, which did not involve any of the classical endocytic proteins (clathrin, dynamin, the actin cytoskeleton), has recently been described [17]. This type of "excessive" endocytosis, which might affect up to $25 \%$ of a cell's surface [17] reflects the formation of ceramide domains that develop high inward curvature and undergo spontaneous budding [41]. This phenomenon is probably analogous to the internalization of ceramide platforms visualized in our previous work [18]. Our present data highlights potential functional consequences which might arise from such a profound reorganisation of the plasmalemma elicited by $\left[\mathrm{Ca}^{2+}\right]_{\mathrm{i}}$ overload.

Proximity to plasmalemmal invagination, as determined by electron tomography was observed for $4.1 \%$ of mitochondria. And though the spatial resolution of this technique can hardly be surpassed, we opted to follow this dynamic process with a higher degree of temporal precision available in the confocal microscope. Plasmalemmal to mitochondrial proximity, as determined by colocalization of the respective markers in the confocal microscopy, occurred for $\sim 30 \%$ of mitochondria, regardless of the cell type or the method selected to induce $\mathrm{Ca}^{2+}$ overload.

Permeabilization of the outer mitochondrial membrane leads to a kinetically invariant, coordinate release of cytochrome c [42]. Yet, though this release was reported to be complete once it commenced, it did not occur simultaneously in all mitochondria, but appeared to affect different groups at different times. Indeed, 

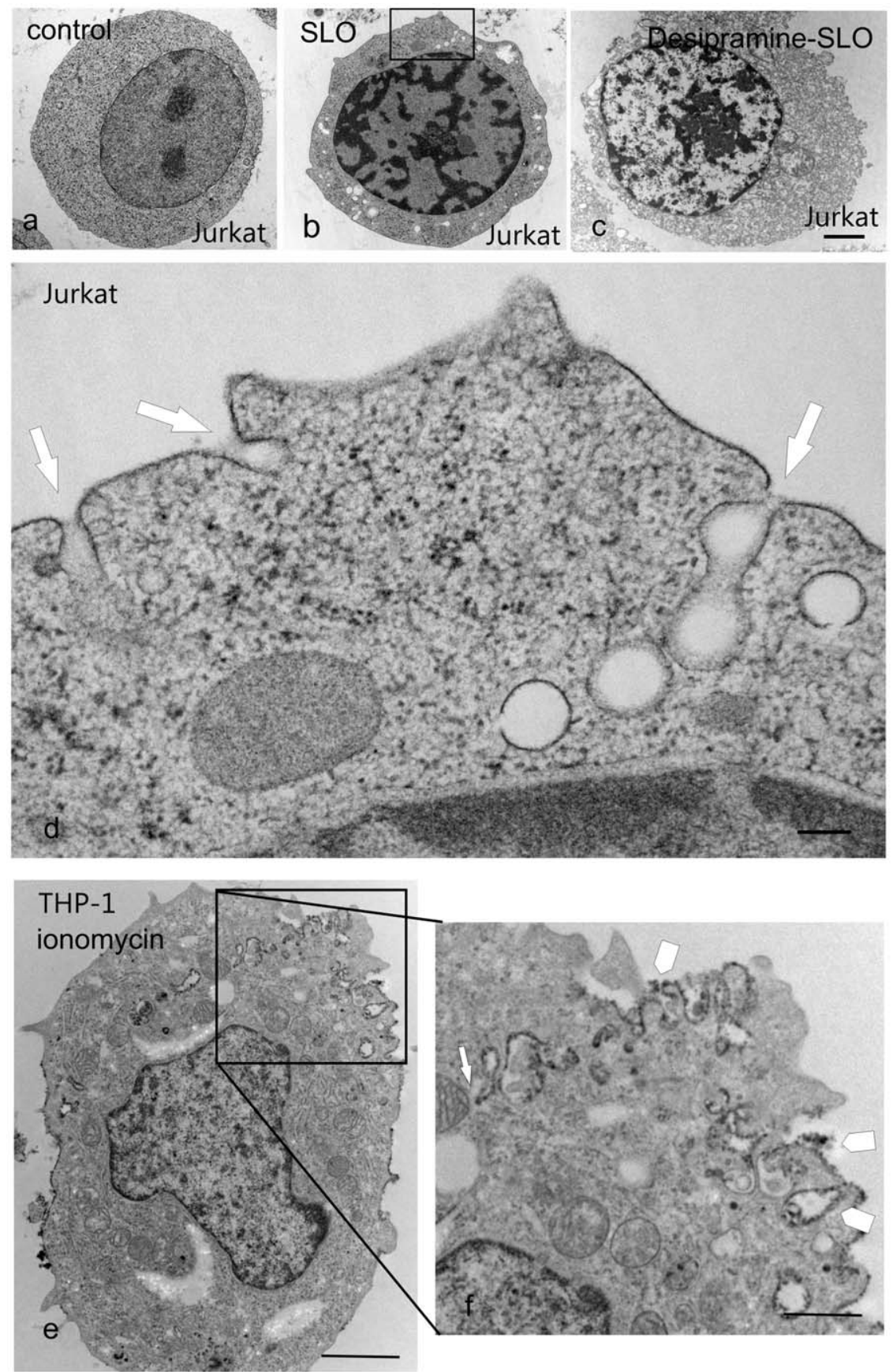

Figure 4. Tube-like invagination of the plasma membrane after intracellular $\mathrm{Ca}^{2+}$-overload. Electron micrographs of unfixed, high pressure frozen, freeze-substituted Jurkat T cells and THP-1 cells at rest (a), after stimulation with SLO (b,d) and after treatment with sphingomyelinase inhibitor desipramine before stimulation with SLO (c). (e,f) THP-1 cells were pre-labelled with horseradish peroxidase-cholera toxin B (arrowheads) before treatment with ionomycin. a: The untreated (control) cell displays a homogeneous cytoplasm and a uniformally smooth plasmalemmal surface. b,d: The SLO-stimulated cell contains numerous cytoplasmic vacuolations, which at higher resolution [d (boxed area in b] turn out to be the profiles of tubular invaginations of the plasma membrane (arrows). c: For the inhibition of sphingomyelinase, the cells were preincubated at $37^{\circ} \mathrm{C}$ for 90 minutes in the presence of $50 \mu \mathrm{M}$ desipramine prior to stimulation with SLO. e: please note deep surface-labelled plasma membrane invaginations, boxed area enlarged in (f, arrow) Bars: $a, b, c, e=1 \mu \mathrm{m}, d=0.2 \mu \mathrm{m}, f=0.5 \mu \mathrm{m}$.

doi:10.1371/journal.pone.0023706.g004

the pattern depicted by Goldstein et al. [42] indicates that mitochondria, which are situated in the immediate vicinity of the plasma membrane are affected earlier than more centrally localized ones.

The close apposition of heterologous membranes has been observed to play a role in the transport of small molecules [43], and molecular markers for the contact sites are currently being identified [44- 46]. Specialized contact sites between the endoplasmic reticulum (ER) or Golgi apparatus and mitochondria are important in the regulation of $\mathrm{Ca}^{2+}$-homeostasis [47-50]. The intracellular transport of lipids, which does not occur via vesicular routes, depends likewise on highly-selective transfer mechanisms. Accordingly, 

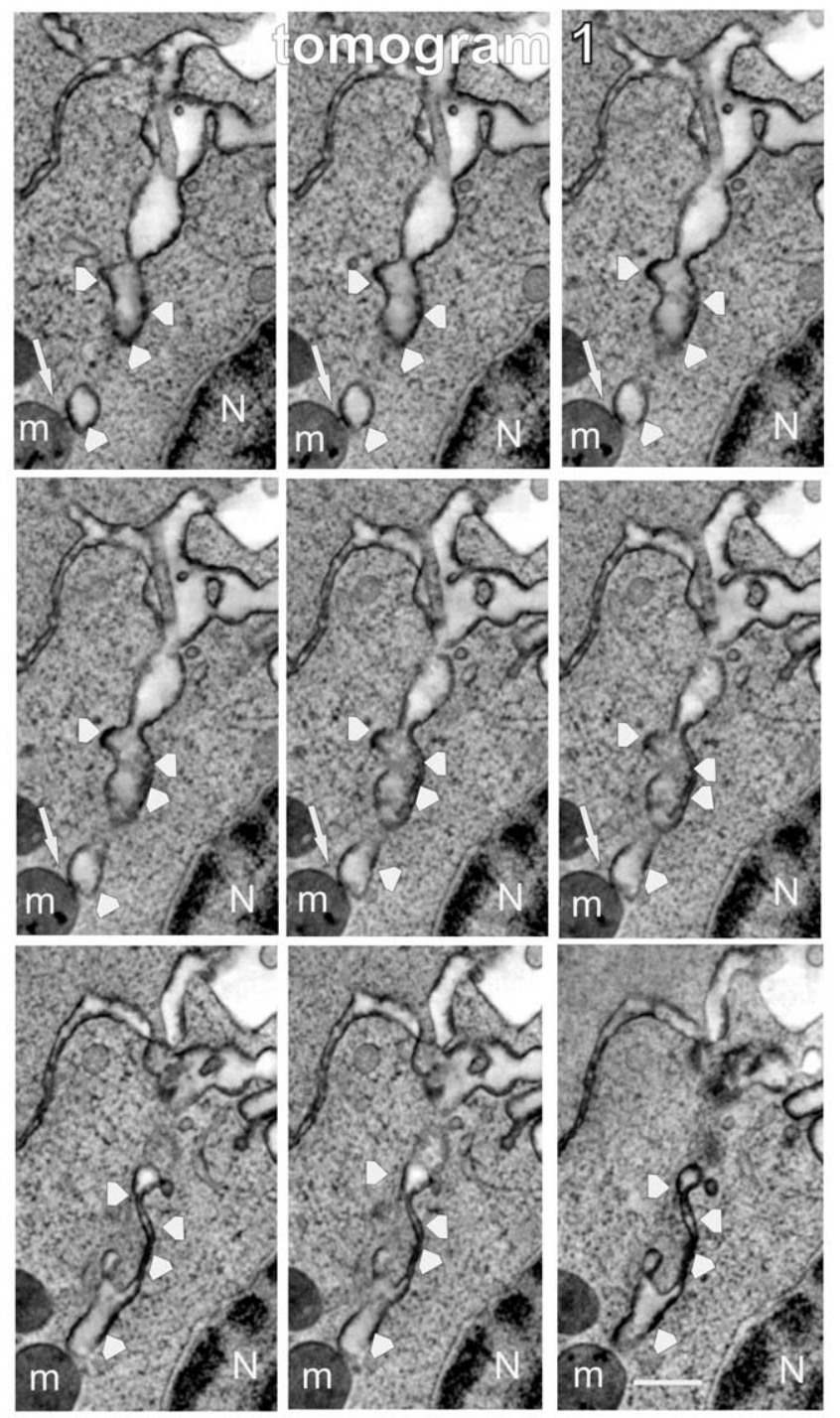
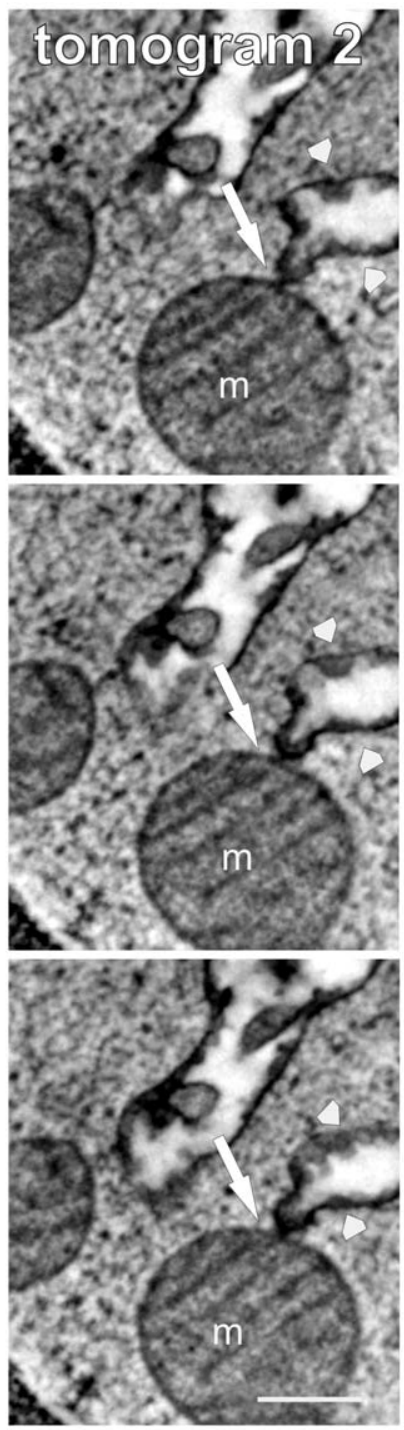

Figure 5. "Kiss-of-death" between the plasma membrane and mitochondria. Movie stills of electron micrographic tomograms of 2 different, unfixed, frozen Jurkat cells, whose outer leaflets of the plasma membrane were pre-labelled with horseradish peroxidase-cholera toxin B (arrowheads) before subjecting the cells to $\mathrm{Ca}^{2+}$ overload. Contact sites between surface-labelled membrane invaginations which extend deeply into the cytoplasm and mitochondrial outer membranes are visible (arrows). Mitochondrium (m), Nucleus (N). Bars $=0.5 \mu \mathrm{m}$. doi:10.1371/journal.pone.0023706.g005

phosphatidylserine is transferred from the ER to the mitochondria via specific, mitochondrion-associated ER-membrane sites [51]. In the case of ceramide, both vesicular and non-vesicular shuttles between the ER and the Golgi apparatus have been described [52,53]. The non-vesicular transport is mediated by the ceramide transfer protein (CERT) and depends upon the integrity of Golgi contact sites [54]. The direct contacts that we observed between the plasma membrane and the mitochondria described here highlights the role of interorganellar contacts in the transport of signalling molecules.

A murine mitochondria-associated neutral sphingomyelinase has been recently identified [10]. Interestingly, this enzyme is activated by phosphatidylserine [10], which is highly abundant on the inner leaflet of the plasma membrane. It is thus conceivable that - in addition to a direct transfer of ceramide - the phosphatidylserine-dependent activation of a mitochondriallyassociated sphingomyelinase might trigger a local production of ceramide thereby permitting an efficient permeabilization of the outer mitochondrial membrane.
In conclusion, we have observed an accumulation of ceramide within the mitochondria of apoptotic cells and shown that ceramide platforms originating within the plasma membrane are being internalised and come into contact with the mitochondrial outer membrane. It is conceivable that ceramide is being exchanged at these contact points to trigger the release of proapoptotic molecules.

\section{Supporting Information}

Movie S1 AnnexinA1/ceramide platform dynamics in Jurkat T-cells after $\mathbf{C a}^{2+}$-overload. Time-lapse sequence of confocal microscopical images for a Jurkat T-cell that had been transiently transfected with annexin Al-YFP (yellow) and annexin A6 (blue), prior to stimulation with with SLO. Both annexins initially translocate to the plasma membrane. Thereafter, annexin Al segregates from annexin A6 and coalesces into membrane platforms, which are internalised. The development of finger-like 
plasmalemmal invaginations of annexin Al (arrows) is illustrated in Fig. 3.

(MOV)

Movie S2 "Kiss of Death 1." Electron micrographic tomograms of unfixed, high-pressure frozen, freeze-substituted Jurkat T-cells, whose outer leaflets of the plasma membrane had been pre-labelled with the horseradish peroxidase-tagged cholera toxin B before stimulation with SLO. Sites of physical contact between surface-labelled membranous invaginations and the mitochondrial outer membranes are visible.

(MOV)

Movie S3 "Kiss of Death 2." Electron micrographic tomograms of unfixed, high-pressure frozen, freeze-substituted Jurkat T-cells, whose outer leaflets of the plasma membrane had

\section{References}

1. Bleicken S, Classen M, Padmavathi PV, Ishikawa T, Zeth K, et al. (2010) Molecular details of Bax activation, oligomerization, and membrane insertion. J Biol Chem 285: 6636-6647.

2. Green DR, Kroemer G (2004) The pathophysiology of mitochondrial cell death. Science 305: 626-629.

3. George NM, Targy N, Evans JJ, Zhang L, Luo X (2010) Bax contains two functional mitochondrial targeting sequences and translocates to mitochondria in a conformational change- and homo-oligomerization-driven process. J Biol Chem 285: 1384-1392.

4. Martinez-Abundis E, Correa F, Pavon N, Zazueta C (2009) Bax distribution into mitochondrial detergent-resistant microdomains is related to ceramide and cholesterol content in postischemic hearts. FEBS J 276: 5579-5588.

5. Tait SW, Green DR (2010) Mitochondria and cell death: outer membrane permeabilization and beyond. Nat Rev Mol Cell Biol 11: 621-632.

6. Siskind LJ, Kolesnick RN, Colombini M (2002) Ceramide channels increase the permeability of the mitochondrial outer membrane to small proteins. J Biol Chem 277: 26796-26803.

7. Cutler RG, Mattson MP (2001) Sphingomyelin and ceramide as regulators of development and lifespan. Mech Ageing Dev 122: 895-908.

8. Hannun YA, Obeid LM (2002) The Ceramide-centric universe of lipidmediated cell regulation: stress encounters of the lipid kind. J Biol Chem 277: 25847-25850.

9. Zheng W, Kollmeyer J, Symolon H, Momin A, Munter E, et al. (2006) Ceramides and other bioactive sphingolipid backbones in health and disease: lipidomic analysis, metabolism and roles in membrane structure, dynamics, signaling and autophagy. Biochim Biophys Acta 1758: 1864-1884.

10. Wu BX, Rajagopalan V, Roddy PL, Clarke CJ, Hannun YA (2010) Identification and characterization of murine mitochondria-associated neutral sphingomyelinase (MA-nSMase), the mammalian sphingomyelin phosphodiesterase 5. J Biol Chem 285: 17993-18002.

11. Bionda C, Portoukalian J, Schmitt D, Rodriguez-Lafrasse C, Ardail D (2004) Subcellular compartmentalization of ceramide metabolism: MAM (mitochondria-associated membrane) and/or mitochondria? Biochem J 382: 527-533.

12. Futerman AH, Hannun YA (2004) The complex life of simple sphingolipids. EMBO Rep 5: 777-782.

13. Novgorodov SA, Szulc ZM, Luberto C, Jones JA, Bielawski J, et al. (2005) Positively charged ceramide is a potent inducer of mitochondrial permeabilization. J Biol Chem 280: 16096-16105.

14. Gulbins E, Li PL (2006) Physiological and pathophysiological aspects of ceramide. Am J Physiol Regul Integr Comp Physiol 290: R11-R26.

15. Hanada K (2006) Discovery of the molecular machinery CERT for endoplasmic reticulum-to-Golgi trafficking of ceramide. Mol Cell Biochem 286: 23-31.

16. Deng X, Yin X, Allan R, Lu DD, Maurer CW, et al. (2008) Ceramide biogenesis is required for radiation-induced apoptosis in the germ line of $\mathrm{C}$. elegans. Science 322: 110-115.

17. Lariccia V, Fine M, Magi S, Lin MJ, Yaradanakul A, et al. (2011) Massive calcium-activated endocytosis without involvement of classical endocytic proteins. J Gen Physiol 137: 111-132.

18. Babiychuk EB, Monastyrskaya K, Draeger A (2008) Fluorescent annexin Al reveals dynamics of ceramide platforms in living cells. Traffic 9: 1757-1775.

19. Fine M, Llaguno MC, Lariccia V, Lin MJ, Yaradanakul A, et al. (2011) Massive endocytosis driven by lipidic forces originating in the outer plasmalemmal monolayer: a new approach to membrane recycling and lipid domains. J Gen Physiol 137: 137-154.

20. Hilgemann DW, Fine M (2011) Mechanistic analysis of massive endocytosis in relation to functionally defined surface membrane domains. J Gen Physiol 137: $155-172$.

21. Cowart LA, Szulc Z, Bielawska A, Hannun YA (2002) Structural determinants of sphingolipid recognition by commercially available anti-ceramide antibodies. J Lipid Res 43: 2042-2048. been pre-labelled with the horseradish peroxidase-tagged cholera toxin B before stimulation with SLO. Sites of physical contact between surface-labelled membranous invaginations and the mitochondrial outer membranes are visible.

(MOV)

\section{Acknowledgments}

We thank Dr Sarah Potez for critically reading this manuscript and Andrea Hostettler and Barbara Krieger for technical assistance.

\section{Author Contributions}

Conceived and designed the experiments: EB KM AD. Performed the experiments: EB AA KM AD DS CA CB. Analyzed the data: EB AD. Wrote the paper: AD.

22. Monastyrskaya K, Babiychuk EB, Hostettler A, Rescher U, Draeger A (2007) Annexins as intracellular calcium sensors. Cell Calcium 41: 207-219.

23. Babiychuk EB, Draeger A (2000) Annexins in cell membrane dynamics: Ca2+regulated association of lipid microdomains. J Cell Biol 150: 1113-1123.

24. Studer D, Graber W, Al Amoudi A, Eggli P (2001) A new approach for cryofixation by high-pressure freezing. J Microsc 203: 285-294.

25. Studer D, Humbel BM, Chiquet M (2008) Electron microscopy of high pressure frozen samples: bridging the gap between cellular ultrastructure and atomic resolution. Histochem Cell Biol 130: 877-889.

26. Jayadev S, Barrett JC, Murphy E (2000) Elevated ceramide is downstream of altered calcium homeostasis in low serum-induced apoptosis. Am J Physiol Cell Physiol 279: C1640-C1647.

27. Pinton P, Giorgi C, Siviero R, Zecchini E, Rizzuto R (2008) Calcium and apoptosis: ER-mitochondria $\mathrm{Ca} 2+$ transfer in the control of apoptosis. Oncogene 27: 6407-6418.

28. Morales A, Colell A, Mari M, Garcia-Ruiz C, Fernandez-Checa JC (2004) Glycosphingolipids and mitochondria: role in apoptosis and disease. GlycoconjJ 20: 579-588.

29. Babiychuk EB, Monastyrskaya K, Potez S, Draeger A (2009) Intracellular $\mathrm{Ca}(2+)$ operates a switch between repair and lysis of streptolysin $\mathrm{O}$-perforated cells. Gell Death Differ 16: 1126-1134.

30. Delgado A, Casas J, Llebaria A, Abad JL, Fabrias G (2006) Inhibitors of sphingolipid metabolism enzymes. Biochim Biophys Acta 1758: 1957-1977.

31. Hannun YA, Obeid LM (2008) Principles of bioactive lipid signalling: lessons from sphingolipids. Nat Rev Mol Cell Biol 9: 139-150.

32. Pacher P, Hajnoczky G (2001) Propagation of the apoptotic signal by mitochondrial waves. EMBO J 20: 4107-4121.

33. Ganesan V, Perera MN, Colombini D, Datskovskiy D, Chadha K, et al. (2010) Ceramide and activated Bax act synergistically to permeabilize the mitochondrial outer membrane. Apoptosis 15: 553-562.

34. Andrieu-Abadie N, Levade T (2002) Sphingomyelin hydrolysis during apoptosis. Biochim Biophys Acta 1585: 126-134.

35. Gulbins E, Kolesnick R (2003) Raft ceramide in molecular medicine. Oncogene 22: 7070-7077.

36. Kirschnek S, Paris F, Weller M, Grassme H, Ferlinz K, et al. (2000) CD95mediated apoptosis in vivo involves acid sphingomyelinase. J Biol Chem 275: 27316-27323.

37. Grassme H, Kirschnek S, Riethmueller J, Riehle A, von KG, et al. (2000) CD95/CD95 ligand interactions on epithelial cells in host defense to Pseudomonas aeruginosa. Science \%20;290: 527-530.

38. Paris F, Grassme H, Cremesti A, Zager J, Fong Y, et al. (2001) Natural ceramide reverses Fas resistance of acid sphingomyelinase $(-/-)$ hepatocytes. J Biol Chem 276: 8297-8305.

39. Verdurmen WP, Thanos M, Ruttekolk IR, Gulbins E, Brock R (2010) Cationic cell-penetrating peptides induce ceramide formation via acid sphingomyelinase: Implications for uptake. J Control Release.

40. Rensing KH, Samuels AL, Savidge RA (2002) Ultrastructure of vascular cambial cell cytokinesis in pine seedlings preserved by cryofixation and substitution. Protoplasma 220: 39-49.

41. Goni FM, Alonso A (2009) Effects of ceramide and other simple sphingolipids on membrane lateral structure. Biochim Biophys Acta 1788: 169-177.

42. Goldstein JC, Waterhouse NJ, Juin P, Evan GI, Green DR (2000) The coordinate release of cytochrome c during apoptosis is rapid, complete and kinetically invariant. Nat Cell Biol 2: 156-162.

43. Levine $\mathrm{T}$ (2004) Short-range intracellular trafficking of small molecules across endoplasmic reticulum junctions. Trends Cell Biol 14: 483-490.

44. de Brito OM, Scorrano L (2010) An intimate liaison: spatial organization of the endoplasmic reticulum-mitochondria relationship. EMBO J 29: 2715-2723.

45. de Brito OM, Scorrano L (2008) Mitofusin 2 tethers endoplasmic reticulum to mitochondria. Nature 456: 605-610. 
46. Kornmann B, Currie E, Collins SR, Schuldiner M, Nunnari J, et al. (2009) An ER-mitochondria tethering complex revealed by a synthetic biology screen. Science 325: 477-481.

47. Rizzuto R, Pinton P, Carrington W, Fay FS, Fogarty KE, et al. (1998) Close contacts with the endoplasmic reticulum as determinants of mitochondrial $\mathrm{Ca} 2+$ responses. Science 280: 1763-1766.

48. Dolman NJ, Gerasimenko JV, Gerasimenko OV, Voronina SG, Petersen OH, et al. (2005) Stable Golgi-mitochondria complexes and formation of Golgi $\mathrm{Ca}(2+)$ gradients in pancreatic acinar cells. J Biol Chem 280: 15794-15799.

49. Csordas G, Varnai P, Golenar T, Roy S, Purkins G, et al. (2010) Imaging interorganelle contacts and local calcium dynamics at the ER-mitochondrial interface. Mol Cell 39: 121-132.
50. Csordas G, Renken G, Varnai P, Walter L, Weaver D, et al. (2006) Structural and functional features and significance of the physical linkage between ER and mitochondria. J Cell Biol 174: 915-921.

51. Vance JE (1991) Newly made phosphatidylserine and phosphatidylethanolamine are preferentially translocated between rat liver mitochondria and endoplasmic reticulum. J Biol Chem 266: 89-97.

52. Funato K, Riezman H (2001) Vesicular and nonvesicular transport of ceramide from ER to the Golgi apparatus in yeast. J Cell Biol 155: 949-959.

53. Hanada K, Kumagai K, Yasuda S, Miura Y, Kawano M, et al. (2003) Molecular machinery for non-vesicular trafficking of ceramide. Nature 426: 803-809.

54. Hanada K, Kumagai K, Tomishige N, Yamaji T (2009) CERT-mediated trafficking of ceramide. Biochim Biophys Acta 1791: 684-691. 